\title{
The Ramsey Type Model with Endogenous Birth Rate
}

\section{Donghan Cai ${ }^{1, \text { a }}$ Jun Wang ${ }^{1}$, Huan Yang ${ }^{1}$, Changyan Zhu ${ }^{1}$}

${ }^{1}$ College of Mathematics and Statistics, Wuhan University, Wuhan, 430072, China

aemail: dhcai@whu.edu.cn

Keywords: Ramsey model, birth rate, equilibrium, phase diagram analysis.

\begin{abstract}
In this paper, a Ramsey type model with endogenous birth rate is set up to inquire the optimal birth rate choosing in the economic growth process. It is proved the model has a unique equilibrium which is saddle under some given conditions. Along the optimal growth path, the per capita capital, consumption and the birth rate increase. By comparative static analysis, it obtain the per capita consumption decreases with the life expectancy prolonged.
\end{abstract}

\section{Introduction}

In recent year, the relationship between population aging and economic growth arouses much attention of economist and demographer [1] [2] [3] [4] [5]. In the theoretic analysis framework, population aging causes the change of demographic structure and economic growth which is also studied by economist and demographer [6] [7] [8] [9].

In the paper [1], Prettner introduces endogenous birth rate into Blanchard's model [10] to investigate the consequences of population aging for long-run economic growth perspectives. In the model, the individuals face a constant age independent instantaneous risk of death which is denoted by $\mu$ and obtain utility from both consumption and birth rate. So, the individual maximizes his or her discounted stream of lifetime utility.

$$
u=\int_{t_{0}}^{\infty} e^{-(\rho+\mu)\left(\tau-t_{0}\right)}[\log (c)+\gamma \log (\beta)] \mathrm{d} \tau,
$$

subject to

$$
\dot{k}=(r+\mu-\delta) k+\hat{w}-(1+\psi \beta) c .
$$

In this paper, by using a Ramsey type model, we modify this model from two aspects. Firstly, the utility function from the consumption and birth rate are both the constant intertemporal elasticity of substitution. Secondly, the production function is neoclassical and the market is perfect competition. It is proved the model has a unique equilibrium which is saddle under some given conditions. Along the optimal growth path, the per capita capital, consumption and the birth rate increase. By comparative static analysis, it obtain the per capita consumption decreases with the life expectancy prolonged.

\section{Setup the Model}

Assume that the intensive production function $f(k)$ satisfies the Inada conditions, i.e.,

$$
f(0)=0, f^{\prime}(k)>0, f^{\prime \prime}(k)<0, \lim _{k \rightarrow 0^{+}} f(k)=+\infty, \lim _{k \rightarrow+\infty} f(k)=0 \text {. }
$$

And the market is perfect competition, $r=f^{\prime}(k)$ and $\hat{w}=f(k)-k f^{\prime}(k)$. The utility function of individual from the consumption and birth rate is strong additive, i.e., $u(c, \beta)=u_{1}(c)+u_{2}(\beta)$.

$$
\begin{aligned}
& \max \int_{0}^{\infty} e^{-(\rho+\mu) t}\left[u_{1}(c)+u_{2}(\beta)\right] \mathrm{d} t, \\
& \text { s.t. } \dot{k}=f(k)-(1+\psi \beta) c-(\delta-\mu) k .
\end{aligned}
$$

The current value Hamiltonian to solve the optimization is

$$
H=u_{1}(c)+u_{2}(\beta)+\lambda[f(k)-(1+\psi \beta) c-(\delta-\mu) k] .
$$

The first order conditions and transversality condition are 


$$
\begin{aligned}
& \dot{k}=\frac{\partial H}{\partial \lambda}=f(k)-(1+\psi \beta) c-(\delta-\mu) k, \\
& \dot{\lambda}=(\rho+\mu) \lambda-\frac{\partial H}{\partial k}=\left[\rho+\mu-f^{\prime}(k)+(\delta-\mu)\right] \lambda, \\
& 0=\frac{\partial H}{\partial c}=u_{1}(c)-(1+\psi \beta) \lambda, \\
& 0=\frac{\partial H}{\partial \beta}=u_{2}(\beta)-\psi c \lambda, \\
& 0=\lim _{t \rightarrow \infty} e^{-(\rho+\mu) t} \lambda(t) k(t),
\end{aligned}
$$

From (8) and (9)

$\psi c u_{1}(c)=u_{2}(\beta)(1+\psi \beta)$.

Lemma 1. Let $g(\beta)=u_{2}(\beta)(1+\psi \beta)$ and satisfy $\lim _{\beta \rightarrow 0} g(\beta)=+\infty, \lim _{\beta \rightarrow \infty} g(\beta)=0$ and $g^{\prime}(\beta)<0$, the equation (11) determines a function $\beta=h(c)$.

Remark 1. When $u_{2}(\beta)$ is the constant intertemporal elasticity of substitution utility function $\frac{\beta^{1-\theta_{2}}-1}{1-\theta_{2}}$ with $\theta_{2}>1$, the conditions of Lemma 1 hold. In fact, $g(\beta)=\beta^{-\theta_{2}}(1+\psi \beta)$ and $g^{\prime}(\beta)=-\beta^{-\theta_{2}-1}\left[\theta_{2}+\left(\theta_{2}-1\right) \psi \beta\right]<0$ in this case.

Remark 2. If the utility function with respective with consumption is the constant intertemporal elasticity of substitution utility function $\frac{c^{1-\theta_{1}}-1}{1-\theta_{1}}$ with $\theta_{1}>1$, then $\lim _{c \rightarrow 0^{+}} h(c)=0, \lim _{c \rightarrow+\infty} h(c)=+\infty$. In fact, from $\psi c u_{1}(c)=\psi c^{1-\theta_{1}}, \lim _{c \rightarrow 0^{+}} \psi c u_{1}(c)=+\infty, \lim _{c \rightarrow+\infty} \psi c u_{1}(c)=0$ and Remark 1, $\lim _{c \rightarrow 0^{+}} h(c)=0, \quad \lim _{c \rightarrow 0^{+}} h(c)=0, \lim _{c \rightarrow+\infty} h(c)=+\infty$ in this case. By (8),

$\ln \lambda+\ln (1+\psi h(c))=\ln u_{1}(c)$.

Taking derivative with respect to time $t$ on the both side of above equation, we have $\frac{\dot{\lambda}}{\lambda}+\frac{\psi h^{\prime}(c)}{1+\psi h(c)} \dot{c}=\frac{u_{1}(c)}{u_{1}(c)} \dot{c}$,

that is,

$$
\left[\frac{\psi h^{\prime}(c)}{1+\psi h(c)}-\frac{u_{1}(c)}{u_{1}(c)}\right] \dot{c}=-\frac{\dot{\lambda}}{\lambda} .
$$

Denote $\frac{\psi h^{\prime}(c)}{1+\psi h(c)}-\frac{u_{1}(c)}{u_{1}(c)}$ by $h_{1}(c)$, then, from (7), we obtain

$$
h_{1}(c) \dot{c}=f^{\prime}(k)-(\rho+\delta) \text {. }
$$

Let $S=\left\{c \mid h_{1}(c) \neq 0\right\}$, then we get following dynamical system

$$
\begin{aligned}
& \dot{k}=f(k)-[1+\psi h(c)] c-(\delta-\mu) k, \\
& \dot{c}=\frac{1}{h_{1}(c)}\left[f^{\prime}(k)-(\rho+\delta)\right] .
\end{aligned}
$$

on the open set $E=R^{+} \times S$.

Remark 3. If the utility function with respective with consumption is the constant intertemporal elasticity of substitution utility function $\frac{c^{1-\theta_{1}}-1}{1-\theta_{1}}$ with $\theta_{1}>1$, then from (11), $h^{\prime}(c)=\frac{\mathrm{d} \beta}{\mathrm{d} c}=c \psi\left[u_{1}(c)+c u_{1}(c)\right] g^{\prime}(\beta)=\frac{\psi\left(1-\theta_{1}\right)}{c^{\theta_{1}} g^{\prime}(\beta)}>0$ with the utility function with respective with birth rate. Therefore, $h_{1}(c)>0$ and $E=R_{2}^{+}$since $-\frac{u_{1}(c)}{u_{1}(c)}=\frac{\theta_{1}}{c}>0$.

Theorem 1. If the utility function with respective with consumption and birth rate are both the constant intertemporal elasticity of substitution with the substitution $0<\sigma_{i}=\frac{1}{\theta_{i}}<1, i=1,2$, then the 
dynamics of the model is described by the dynamical system (14)-(15).

\section{The Existence and Uniqueness of the Non-zero Equilibrium}

In this section, we assume that utility functions satisfy the condition of Theorem 1.

Theorem 2. The dynamical system (14)-(15) has a unique non-zero equilibrium.

Proof. Since $\lim _{k \rightarrow 0^{+}} f^{\prime}(k)=+\infty, \lim _{k \rightarrow+\infty} f^{\prime}(k)=0$ and $f^{\prime \prime}(k)<0$, there exists a unique $k^{*}>0$ such that $f^{\prime}\left(k^{*}\right)=\rho+\delta$.

Let $h_{2}(c)=[1+\psi h(c)] c$, then, from

$h_{2}(c)=1+\psi h(c)+h^{\prime}(c) c>0$, and $\lim _{c \rightarrow 0^{+}} h_{2}(c)=0, \lim _{c \rightarrow+\infty} h_{2}(c)=+\infty$

there exists a unique $c^{*}$ such that $f\left(k^{*}\right)-\left[1+\psi h\left(c^{*}\right)\right] c^{*}-(\delta-\mu) k^{*}=0$ for

$f\left(k^{*}\right)-(\delta-\mu) k^{*}=k^{*}\left[\frac{f\left(k^{*}\right)}{k^{*}}-(\delta-\mu)\right]>k^{*}\left[f^{\prime}\left(k^{*}\right)-(\delta-\mu)\right]=k^{*}(\rho+\mu)>0$.

This completes the proof of the Theorem.

Theorem 3. The unique equilibrium $\left(k^{*}, c^{*}\right)$ of the dynamical system (14)-(15) is saddle.

Proof. Let

$$
\begin{aligned}
& F(k, c)=f(k)-[1+\psi h(c)] c-(\delta-\mu) k, \\
& G(k, c)=\frac{1}{h_{1}(c)}\left[f^{\prime}(k)-(\rho+\delta)\right],
\end{aligned}
$$

then the Jacobian of the dynamical system (14)-(15) at the equilibrium is

$$
J=\left[\begin{array}{ll}
\frac{\partial F}{\partial k} & \frac{\partial F}{\partial c}(18) \\
\frac{\partial G}{\partial k} & \frac{\partial G}{\partial c}
\end{array}\right]_{\left(k^{*}, c^{*}\right)}=\left[\begin{array}{cc}
\rho+\mu & -\left[1+\psi h\left(c^{*}\right)+h^{\prime}\left(c^{*}\right) c^{*}\right] \\
\frac{f^{\prime \prime}\left(k^{*}\right)}{h_{1}\left(c^{*}\right)} & 0
\end{array}\right]
$$

Denote $-\left[1+\psi h\left(c^{*}\right)+h^{\prime}\left(c^{*}\right) c^{*}\right]$ and $\frac{f^{\prime \prime}\left(k^{*}\right)}{h_{1}\left(c^{*}\right)}$ by $a_{2}$ and $a_{3}$, then $a_{2}<0, a_{3}<0$, and the characteristic equation and eigenvalues of the matrix $J$ are

$$
\begin{aligned}
& v^{2}-(\rho+\mu) v-a_{2} a_{3}=0, \\
& v_{1,2}=\frac{1}{2}\left[\mu+\rho \pm \sqrt{(\rho+\mu)^{2}+4 a_{2} a_{3}}\right] .
\end{aligned}
$$

The eigenvalues $v_{1}, v_{2}$ are real and have opposite sign since $a_{2} a_{3}>0$. Therefore, the unique equilibrium of the dynamical system (14)-(15) is saddle and the Theorem holds.

\section{Phase Diagram Analysis}

Lemma 2. If $\delta>\mu$, then the equation $F(k, c)=0$ determines a curve $c_{1}(k)$ such that $F\left(k, c_{1}(k)\right)=0$ on the interval $\left[0, k_{1}\right]$, where $k_{1}>k^{*}$ is the unique positive solution of the equation $f(k)-(\delta-\mu) k=0$.

Proof. Since $\lim _{k \rightarrow 0^{+}} \frac{f(k)}{k}=+\infty, \lim _{k \rightarrow+\infty} \frac{f(k)}{k}=0$ and $\frac{\mathrm{d}}{\mathrm{d} k} \frac{f(k)}{k}<0$, the equation $f(k)-(\delta-\mu) k=0$ has a unique positive solution $k_{1}$.

From the proof of Theorem 2, $h_{2}(c)$ strictly increases from zero to positive infinity. Hence, $c_{1}(k)=h_{2}^{-1}[f(k)-(\delta-\mu) k]$ on the interval and $c_{1}(0)=c_{1}\left(k_{1}\right)=0$. This completes the proof of Lemma.

By the proof of Theorem 2 and Lemma 2, we see that the loci of $\dot{k}=0$ is the line $k=k^{*}$ and the loci of $\dot{c}=0$ is the inverted U-shaped curve $c_{1}(k)$ on the first quadrant of $k-c$ plane. The 
line $k=k^{*}$ and the curve $c_{1}(k)$ divide the first quadrant of $k-c$ plane into four parts and we have following phase diagram(See figure 1).

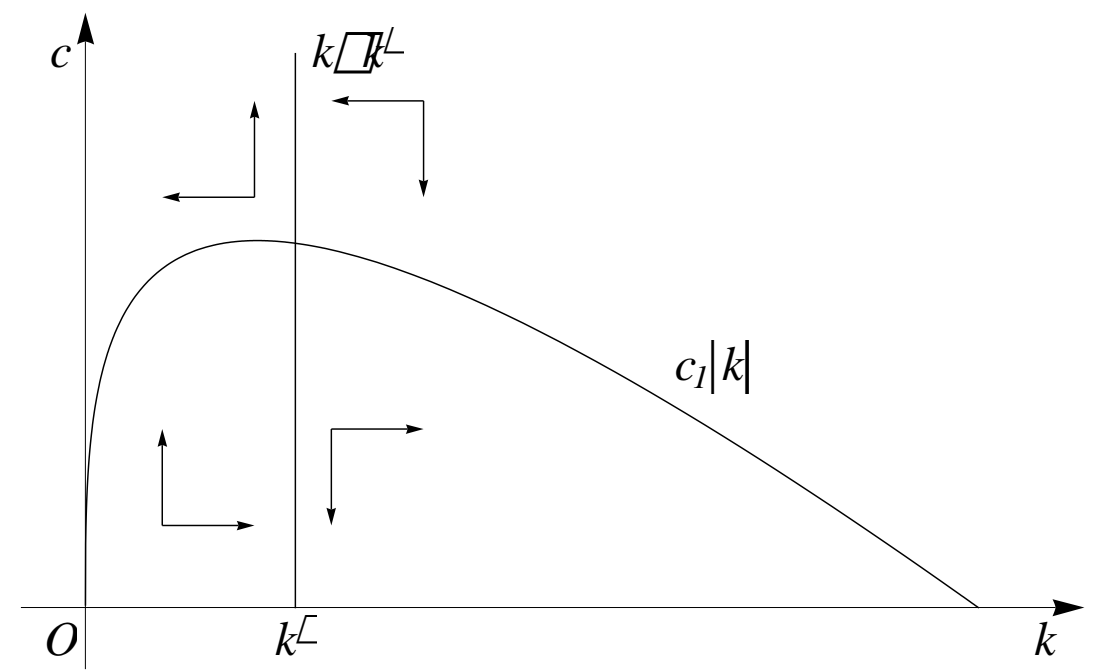

Fig.1: The diagram of the dynamical system

From phase diagram analysis and $\frac{\mathrm{d} \beta}{\mathrm{d} c}=h^{\prime}(c)>0$, we have following Theorem

Theorem 4. The economy which described by the model has optimal growth path on which the per capita capital, per capital consumption and the birth rate strictly increase.

\section{The effect of the Longevity on Economic Growth}

From the proof of Theorem 3, we see that the per capita capital $k^{*}$ is independent the parameter $\mu$, which implies the death rate $\mu$ does not effect the per capita capital at steady state.

Let $Q\left(c^{*}, \mu\right)=f\left(k^{*}\right)-\left[1+\psi h\left(c^{*}\right)\right] c^{*}-(\delta-\mu) k^{*}$, then from, $\frac{\mathrm{d} c^{*}}{\mathrm{~d} \mu}=-\frac{\frac{\partial Q}{\partial c^{*}}}{\frac{\partial Q}{\partial \mu}}=\frac{h_{2}\left(c^{*}\right)}{k^{*}}>0$ and the life expectancy is $\frac{1}{\mu}^{[10]}$, we have following theorem

Theorem 5. The per capita consumption at steady state increases with the death rate increasing and decreases with population aging.

\section{Conclusion}

In the section 2, we first obtain the dynamical system which described the model under given conditions by solving the optimization problem. The existence and uniqueness of non-zero equilibrium of the dynamical system and the unique equilibrium is saddle has been proved in the section 3. By phase diagram analysis, it is proved the model has optimal growth path in the section 4. In the section 5, the affect of population on the economic growth is studied by comparative static analysis and obtained that per capita capital consumption decreases with population aging.

\section{Acknowledgement}

In this paper, the research was sponsored by the Nature Science Foundation of China(Project No. 71271158).

\section{References}

[1] Klaus Prettner. Population aging and endogenous economic growth[J]. Journal of Population Economy, 26(2013): 811-834 
[2] Mehmet Serkan Tosun. Population aging and economic growth: political economy and open economy effects[J]. Economics Letters, 81(2003): 291-296

[3] Maxime Fougère, Simon Harvey, Jean Mercenier, Marcel Mérette M. Population ageing, time allocation and human capital: A general equilibrium analysis for Canada[J]. Economic Modelling, 26(2009): 30-39

[4] David E. Bloom, David Canning, Günther Fink. Implications of population ageing for economic growth[J]. Oxford Review of Economic Policy, 26(2010): 583-612

[5] Koichi Futagami, Tetsuya Nakajima. Population aging and economic growth[J]. Journal of Macroeconomics, 23(2001): 31-44

[6] Hippolyte d'Albis. Demographic structure and capital accumulation. Journal of Economic Theory, 132(2007): 411-434

[7] Sau-Him Paul Lau. Demographic structure and capital accumulation: A quantitative assessment[J]. Journal of Economic Dynamics \& Control, 33(2009): 554-567

[8] Heinrich Hock, David N. Weil D N. On the dynamics of the age structure, dependency, and consumption[J]. Journal of Population Economy, 25(2012): 1019-1043

[9] Solveig Erlandsen, Ragnar Nymoen. Consumption and population age structure[J]. Journal of Population Economics, 21(2008): 505-520

[10] Olivier J. Blanchard. Debt, deficits, and finite horizons[J]. Journal of Political Economy, 2(1985): 223-247 